\title{
Anatomical study of the anterior neurovascular interval approach to the elbow: observation of the neurovascular interval and relevant branches
}

\author{
X.-H. Yang' ${ }^{1}$, C. Wei², G.-P. Li' ${ }^{1}$, J.-J. Wang' ${ }^{1}$, H.-T. Zhao', L.-T. Shi', X.-Y. Cao', Y.-Z. Zhang ${ }^{2}$ \\ ${ }^{1}$ Second Department of Orthopaedics, Affiliated Hospital of Chengde Medical College, Chengde, China \\ 2Emergency Centre of Trauma, Third Hospital of Hebei Medical University, Shijiazhuang, China
}

[Received: 31 May 2019; Accepted: 11 July 2019]

\begin{abstract}
Background: The aim of the study was to investigate the anatomy of the anterior nerve and artery of the elbow joint to provide reference on the relevant surgical approach to the elbow joint, and determine a simple better surgical approach for the treatment of part of the fractures of the elbow joint.

Materials and methods: The upper extremities of 10 adult cadavers fixed by formaldehyde and perfused with red latex in the artery were observed to investigate the anatomic structure of the anterior approach to the elbow joint. From the clearance of the brachioradialis and pronator teres muscle to the approach of the neurovascular interval, we observed the states of the median nerve, the brachial, radial and ulnar arteries, and its branches through anatomical layers and measurement methods.

Results: Through the anterior neurovascular interval approach to the elbow, nerve and artery can be protected, and the anterior structures of the elbow, such as the ulna coronoid process, humeroulnar joint and trochlea of the humerus, can be exposed.

Conclusions: This study demonstrates that the anterior anatomical structure of the elbow joint including the trochlea of the humerus, coronoid process of the ulna and the front capsule of the elbow can be exposed through the anterior neurovascular approach to the elbow. (Folia Morphol 2020; 79, 2: 387-394)
\end{abstract}

Key words: anatomy, anterior approach, elbow joint, neurovascular interval

\section{INTRODUCTION}

An anatomical study was conducted on the brachial, radial and ulnar arteries, the concomitant median nerves, as well as related branches, based on the anterior approach to the elbow. Surgical exposures for complex injuries of the elbow are technically demanding due to the high density of neurologic, vascular and ligamentous elements around the elbow. The posterior approach, lateral approach and medial approach are usually applied. Furthermore, these approaches may be combined to address complex pathologies in the setting of fracture fixation, arthroplasty and capsular release [17]. The anterior approach to the elbow is rarely used in clinical practice, but is mainly used for exposing neurovascular structures of the anterior cubital fossa, especially for anterior neurovascular exploration [16]. Due to the necessity to expose different anatomic sites of the elbow, the biceps tendon was used as the boundary to divide the anterior approach into the anterolat-

Address for correspondence: Dr. Y.-Z. Zhang, Emergency Centre of Trauma, Third Hospital of Hebei Medical University, No. 139 of Ziqiang Road, Qiaoxi District, Shijiazhuang 050051, China, tel: +86 311 88603610, fax: +86 31187023626 , e-mail: zhangyingze880923@163.com 
eral and anteromedial approach to the elbow. The anterior approach to the elbow with incision at the lateral biceps tendon is defined as the anterolateral approach to the elbow, while that at the medial biceps tendon is called the anteromedial approach to the elbow. Through the anatomical study of relevant nerves and blood vessels of the anterior elbow, we aimed to provide reference for the study of associated surgical approaches to the elbow, thereby providing novel and simple surgical approaches for the clinical treatment of various fractures of the elbow.

\section{MATERIALS AND METHODS}

Anatomical specimens

A total of 10 left and 10 right extremities of 10 (6 males and 4 females) formalin-fixed adult cadavers and those that were perfused with red latex in the artery were selected for this study. All cadavers had no traumas and deformities, and no degenerative changes in the elbows (provided by the Department of Anatomy, Chengde Medical College).

\section{Experimental apparatus and tools}

- Digital Camera: Canon

- Vernier calliper: Measuring Tools Co., Ltd. (Hangzhou), with an accuracy of $0.02 \mathrm{~mm}$

- Steel ruler

- Limb supporting instruments

- Other commonly used anatomical instruments

\section{Experimental methods}

The "S" type incision started at the anterior elbow, curved cross the elbow crease, and extended distally along the radial border of the forearm. Layer by layer, the dissection was performed at the neurovascular space along the median nerve, brachial artery and ulnar artery. This exposed the joint capsule of the anterior elbow, the coronoid fossa of the humerus, the articular surface of the distal humeral trochlea, the humeral head, the humeroulnar joint and the ulnar coronoid.

Both sides of the elbow were dissected layer by layer. The incision was initiated at biceps tendon by opening biceps aponeurosis, in order to locate the brachial artery and median nerve. The number of vascular branches of the medial brachial artery and the medial ulnar artery was counted. The bifurcation of the brachial artery and ulnar artery served as a marker to record the position of the vascular branches, and measure its diameters. The position of the lateral branches of the brachial and radial artery, the number of branches, vessel diameter, the length of the blood vessels and concomitant nerves without branches, and the distribution of the median nerve and its branches were also documented. Observational data were expressed as mean \pm standard deviation (SD).

Observations (close to the anterior elbow). Each item was measured three times, and the average was used as the final result:

- length of the blood vessels and concomitant nerves without crossing branches;

- location, number and dimension of lateral branches of the brachial and radial artery;

- location, number and dimension of medial branches of the brachial and ulnar artery;

- muscle branches of the median nerve and its distribution.

\section{Ethics approval and consent to participate}

This study was conducted with approval from the Ethics Committee of the Third Hospital of Hebei Medical University. This study was conducted in accordance with the declaration of Helsinki.

\section{Statistical analysis}

SPSS 13.0 software was used for data analysis. Measurement results were expressed as mean \pm SD. Data on the lateral and medial branches of the left and right blood vessels were compared. $\mathrm{P}<0.05$ was considered statistically significant.

\section{RESULTS}

Anatomical study of the anterior approach to the elbow

The "S" type incision started at $2 \mathrm{~cm}$ proximal to the elbow crease, curved across the elbow crease, and extended distally along the radial border of the forearm approximately $4 \mathrm{~cm}$ distal to the flexion crease. Subcutaneous tissues were cut layer by layer by performing blunt subcutaneous dissections. The cephalic vein and basilic vein were distributed laterally and medially, respectively; and the median cubital vein was connected in the middle, protecting the medial and lateral cutaneous nerve of the medial and lateral forearm. The deep fascia and layer of the deep fascia were unroofed. Removal of skin and tissues of the superficial fascia provided excellent exposure of the biceps and biceps aponeurosis. Transverse split of the biceps aponeurosis provided the best exposure of the distal brachioradialis and pronator teres, as well 
as the brachial artery and vein and the median nerve proximal to the incision. The median nerve extended distally from the space between the humeral head of the pronator teres muscle and ulnar head. The brachioradialis and pronator teres muscle were separated for distal exposure. The space between the brachial artery and median nerve allowed insertion. The one above was the ulnar collateral artery, which was given off medially by the brachial artery; and the one below was the ulnar recurrent artery, which was given off medially by the ulnar artery. The above two branches were concomitant without neurovascular branches in specimens without perfusion. However, in perfused specimens, 1-2 tiny medical artery branches were visible; which extended to the surface of the brachial muscle. The loose structure at the space led to the ready separation. The brachial and ulnar artery, biceps tendon and brachioradialis were retracted laterally; while the median nerve and pronator teres were retracted medially, providing the best exposure of the brachial muscle. The longitudinal split and retraction of the brachial is provided a good visualisation of the anterior capsule, ulnar coronoid process, and articular surface of the humeral trochlea.

Exposure of the joint capsule of the anterior elbow, brachialis insertion, proximal humerus, coronoid fossa of the humerus, articular surface of the distal humeral trochlea, humeral head, humeroulnar joint, coronoid process of ulna, and proximal ulna

Exposure of the anatomical structure of the elbow: The longitudinal split of the brachial muscle and lateral retraction rendered the exposure of the ulnar joint capsule of the anterior elbow. The joint capsule incision led to the exposure of the anterior articular surface of the trochlea. A periosteal detacher was used to detach the brachialis insertion, namely, the ulnar tuberosity, and the partial joint capsule insertion at the ulna coronoid process, to expose the ulna coronoid process and humeroulnar joint. Generally, the ulnar recurrent artery did not have to be cut off, and moving the retractor upward for lateral retraction can expose the coronoid fossa of the humerus. Further retraction laterally would expose part of the humeral head.

The length of the blood vessels and concomitant nerves without crossing branches: The average length was $6.34 \mathrm{~cm}$ to the left side of the male, and an average of $7.26 \mathrm{~cm}$ to the right side. By contrast, the average length to the left side of the female was $5.01 \mathrm{~cm}$, and the average was $5.53 \mathrm{~cm}$ to the right. The length
Table 1. The length of blood vessels and concomitant nerve without branches across them (cm, with two decimal places)

\begin{tabular}{ccccc}
\hline \multicolumn{2}{c}{ Male $(\mathbf{n}=\mathbf{6})$} & & \multicolumn{2}{c}{ Female $(\mathbf{n}=\mathbf{4})$} \\
\cline { 1 - 2 } \cline { 5 - 5 } Left side & Right side & & Left side & Right side \\
\hline 8.50 & 6.34 & & 3.98 & 3.79 \\
5.88 & 8.51 & & 6.56 & 7.63 \\
3.89 & 6.82 & & 4.92 & 4.87 \\
6.82 & 7.68 & & 4.57 & 5.82 \\
7.26 & 7.80 & & & \\
5.68 & 6.42 & & & \\
& & & &
\end{tabular}

$P>0.05$

to the left side was $5.81 \pm 1.50 \mathrm{~cm}$ and the length to the right side was $6.57 \pm 1.45 \mathrm{~cm}$. There was no significant difference when compared with those of the left and right sides $(t=-1.672, p=0.129)$. Furthermore, no significant crossing branches were observed between these; therefore these were not necessary to be cut off and damaged. In addition, lateral retraction was easy. The length provided good exposure to the corresponding parts of the elbow, which has important clinical value (Table 1).

The location, number and dimension of the lateral branches of the brachial and radial artery. Within this area, various vascular branches exist ranging from 2-3 or 3-4. There was a relatively constant branch, namely, the radial recurrent artery, which was dense, thick in diameter and located at the radial and ulnar artery bifurcation or close to the bifurcation. The branch sometimes arose from a trunk and sometimes from several trunks, nourishing the lateral forearm muscles. The diameter of the left radial recurrent artery was $2.88 \pm 0.47 \mathrm{~mm}$ and the diameter of the right radial recurrent artery was $2.79 \pm 0.53 \mathrm{~mm}$, and there was no significant difference when compared with those of the left and right sides $(t=0.499, p=0.630)$. The brachial and ulnar artery had few medial branches, including two constant branches that were $0-1$ at the level of the radial neck, namely, the ulnar recurrent artery. The diameter of the left ulnar recurrent artery was $1.17 \pm 0.77 \mathrm{~mm}$ and the diameter of the right ulnar recurrent artery was $0.99 \pm 0.76 \mathrm{~mm}$, and there was no significant difference when compared with those of the left and right sides ( $t=0.651, p=0.531)$. The other branch was located far away from the radial neck, namely, the ulnar collateral artery. Both were located far apart and were smaller in diameter. These two vascular branches were vertical to the trunk, which is of guiding significance in the direction of 
Table 2. Distance (cm, to the radial, ulnar artery bifurcation) of main branches of brachial and radial artery (radial recurrent artery), and vessel diameter ( $\mathrm{mm})$

\begin{tabular}{|c|c|c|c|c|c|c|c|}
\hline \multicolumn{4}{|c|}{ Male $(n=6)$} & \multicolumn{4}{|c|}{ Female $(n=4)$} \\
\hline \multicolumn{2}{|c|}{ Left side } & \multicolumn{2}{|c|}{ Right side } & \multicolumn{2}{|c|}{ Left side } & \multicolumn{2}{|c|}{ Right side } \\
\hline $\begin{array}{l}\text { Dis- } \\
\text { tance }\end{array}$ & $\begin{array}{l}\text { Diam- } \\
\text { eter }\end{array}$ & $\begin{array}{c}\text { Dis- } \\
\text { tance }\end{array}$ & $\begin{array}{l}\text { Diam- } \\
\text { eter }\end{array}$ & $\begin{array}{c}\text { Dis- } \\
\text { tance }\end{array}$ & $\begin{array}{l}\text { Diam- } \\
\text { eter }\end{array}$ & $\begin{array}{l}\text { Dis- } \\
\text { tance }\end{array}$ & $\begin{array}{c}\text { Diam- } \\
\text { eter }\end{array}$ \\
\hline 0.50 & 3.50 & 0 & 3.50 & 1.00 & 3.00 & 0 & 2.50 \\
\hline 0 & 3.50 & 1.00 & 3.00 & 0 & 2.80 & 0 & 2.60 \\
\hline 0 & 2.50 & 0 & 2.00 & 0 & 2.00 & 0.20 & 3.00 \\
\hline 1.00 & 3.00 & 0.80 & 2.00 & 0.30 & 2.50 & 0 & 2.80 \\
\hline 0 & 3.20 & 0 & 3.50 & & & & \\
\hline 0 & 2.80 & 0.5 & 3.00 & & & & \\
\hline
\end{tabular}

$P>0.05$

Table 3. Distance (cm, to the radial, ulnar artery bifurcation) of main branches of brachial and ulnar artery (ulnar recurrent artery), and vessel diameter (mm)

\begin{tabular}{|c|c|c|c|c|c|c|c|}
\hline \multicolumn{4}{|c|}{ Male $(n=6)$} & \multicolumn{4}{|c|}{ Female $(n=4)$} \\
\hline \multicolumn{2}{|c|}{ Left side } & \multicolumn{2}{|c|}{ Right side } & \multicolumn{2}{|c|}{ Left side } & \multicolumn{2}{|c|}{ Right side } \\
\hline $\begin{array}{l}\text { Dis- } \\
\text { tance }\end{array}$ & $\begin{array}{l}\text { Diam- } \\
\text { eter }\end{array}$ & $\begin{array}{c}\text { Dis- } \\
\text { tance }\end{array}$ & $\begin{array}{l}\text { Diam- } \\
\text { eter }\end{array}$ & $\begin{array}{l}\text { Dis- } \\
\text { tance }\end{array}$ & $\begin{array}{l}\text { Diam- } \\
\text { eter }\end{array}$ & $\begin{array}{l}\text { Dis- } \\
\text { tance }\end{array}$ & $\begin{array}{c}\text { Diam- } \\
\text { eter }\end{array}$ \\
\hline 1.50 & 1.00 & 1.30 & 2.50 & 1.20 & 1.00 & 2.00 & 1.50 \\
\hline 0.95 & 0.80 & 2.00 & 0.50 & 1.80 & 0.50 & 1.60 & 0.48 \\
\hline 1.80 & 0.50 & 1.90 & 0.40 & & & & \\
\hline 2.00 & 2.50 & 1.80 & 0.50 & & & & \\
\hline
\end{tabular}

$P>0.05$

retracting blood vessels for the exposure of related anatomic sites (Tables 2, 3; Figs. 1, 2).

The median nerve gave off branches medially at the muscle branch of the elbow, and basically extended medially; in which only one extended laterally in less samples and part of the pronator teres was governed by the muscle branch (Fig. 3). However, all branches of the muscle branches were given off at a minimal acute angle from the trunk, which were almost parallel to the trunk. This is of great importance for intraoperative retraction to expose the operative field, thereby preventing muscle branch damage.

\section{DISCUSSION}

Anatomical study and features of the anterior neurovascular interval approach to the elbow

The anterior approach to the elbow, often referred to as the Henry approach, is located at the lateral

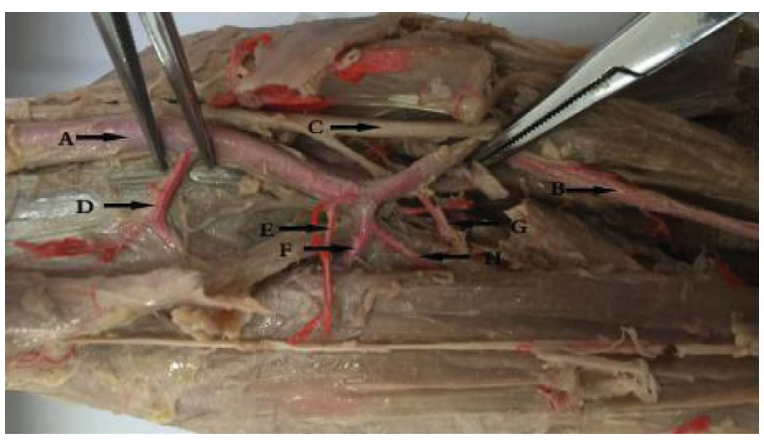

Figure 1. More and thicker lateral branches of brachial and radial artery; $\mathrm{A}$ - brachial artery; $\mathrm{B}$ - radial artery; $\mathrm{C}$ - median nerve; $\mathrm{D}, \mathrm{E}, \mathrm{F}, \mathrm{G}, \mathrm{H}$ - lateral branches of brachial and radial artery.

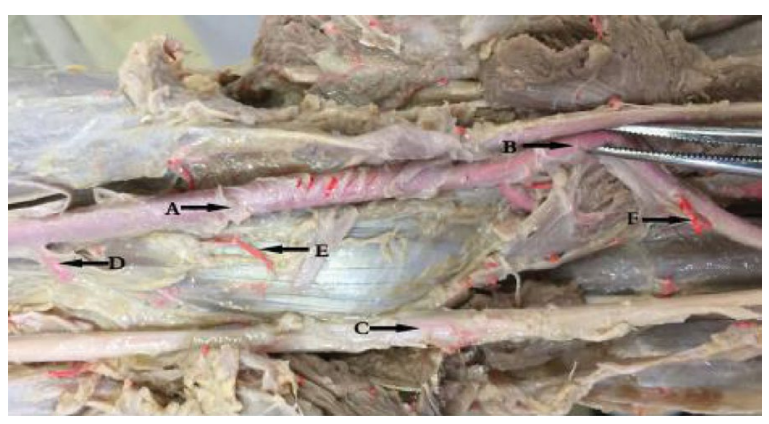

Figure 2. Fewer and thin medial branches of brachial and ulnar artery; A - brachial artery; B - ulnar artery; $\mathrm{C}$ - median nerve; $D, E, F-$ medial branches of brachial and ulnar artery.

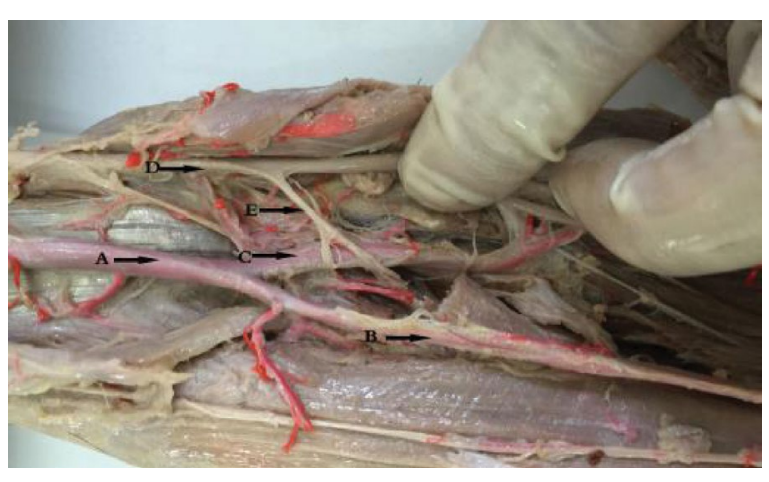

Figure 3. The branch of the median nerve extended laterally; $A-$ brachial artery; $\mathrm{B}$ - radial artery; $\mathrm{C}$ - ulnar artery; D - median nerve; $\mathrm{E}$ - lateral branch of the median nerve.

biceps tendon insertion. In our study, the neurovascular interval approach is located at the medial biceps tendon, technically referred to as the anteromedial approach to the elbow; which presents the exposure of anatomical structures and locations different from the Henry approach. The Henry approach is applied to expose the humeral head, radial head, radial neck and proximal radius; and our approach is more prefer- 
able for exposing the coronary fossa of the humerus, trochlea and ulna coronoid.

The anatomical study in our group revealed the following.

The length of the blood vessels and concomitant nerves without respective branches usually refers to the distance in which the brachial artery and ulnar artery do not give off branches medially, which can be visually observed; and the concomitant median nerves give off no lateral branches in normal cadavers without perfused latex. Under normal circumstances, if no variations exist, median nerves in this section have no branches laterally; which has been confirmed in our anatomical study. However, blood vessels are an exception. Hence, we still found very tiny and short 1-2 medial artery branches in perfused specimens, which extended to the brachial muscle surface. The maximum length of the concomitant vessels was $8.51 \mathrm{~cm}$, and the minimum length was $3.79 \mathrm{~cm}$. This had an average of $6.80 \mathrm{~cm}$ in males, which was longer than in females, with an average of $5.27 \mathrm{~cm}$. This might be correlated with the height, and the branches were not symmetrical on the left and right sides. Most medial vascular branches, which constitute the upper side of arterial network of the elbow, are given off by the ulnar collateral artery from brachial artery; and those constituting the low side are given off by the ulnar recurrent artery.

At the apex of the cubital fossa, the brachial artery divides into its two major branches, the radial and ulnar arteries [8]. The brachial artery and radial artery have more vascular branches laterally, referring to the larger branches of 2-3 or 3-4, most of which are not constant. A relatively constant one, which is dense and thick in diameter, is divided into the radial artery and ulnar artery at the radial and ulnar artery bifurcation, namely, the brachial artery at the level of the radial neck [3], or close to the bifurcation; and sometimes from a trunk and from various trunks. The height is approximately at the level of radial neck, and also at the level of ulna coronoid base, nourishing the lateral forearm muscles. It is impossible to expose the ulna coronoid by retracting the blood vessels medially. Thus, one must cut off the branch vessels, causing great damage. The brachial artery and ulnar artery have a smaller number of larger medial branches. There are two relatively constant ones located far from each other and far from the radial and ulnar artery bifurcation, namely, the coronoid. The distance between the radial branch and the radial and ulnar artery bifurcation was $0.27 \mathrm{~cm}$, which is closer to the coronoid; and the distance to ulnar recurrent artery branch is $1.51 \mathrm{~cm}$, which is convenient for retraction and exposure. Smaller in diameter, these can be cut off and ligated, causing less damage, when it is difficult to extract and expose. Two vessel branches were vertical to the trunk. The radial branch has an averaged diameter of $2.81 \mathrm{~mm}$, and this is significantly greater than the diameter of the ulnar branch, which was $1.03 \mathrm{~mm}$. These two vascular branches that are given off are different [10], which is consistent with this feature. Lateral branches of the brachial and radial artery are closer to the coronoid than to the medial branches of humeral artery and ulnar artery, leading to difficulty in exposure by retraction; and have been confirmed by angiography [14]. The anatomical features of blood vessels in this location has a guiding significance in the direction of retracting blood vessels to expose the anatomical site of the elbow; that is, the lateral retraction of the artery is superior to medial retraction.

Basically, the median nerve gives off branches medially at muscle branches of the elbow, and only one group of muscular branches given off laterally control part of the pronator teres. All muscle branches were given off from the trunk at a minimal acute angle, and were almost parallel to the trunk. The median nerve does not give off branches in the arm, but there are many muscle branches medially in the forearm [7], which is of great significance in intraoperative retraction to expose the operative field, thereby preventing muscle branch damage. Most branches to the muscles in the superficial and intermediate layers of the forearm originate medially from the nerve distal to the elbow joint [8].

\section{The rationality, feasibility and safety of anterior neurovascular interval approach to the elbow to expose the corresponding parts of the elbow}

The anterior approach to the elbow to expose the coronoid, trochlea and anterior joint capsule, namely, the anteromedial part of the elbow, can directly expose the anatomical site. This would facilitate direct visualisation for the operation, which is in line with the principles of the surgical approach to the elbow proposed by scholars [4]. It is reasonable and feasible to expose the coronoid, trochlea and anterior joint capsule. After anatomical studies, the brachial artery, ulnar artery and vein, median nerve and its branches 
are protected from direct visualisation; minimising the risk of neurovascular injury. The incision made at the space among the median nerve, brachial artery and ulnar artery, and lateral retraction of brachioradialis and pronator teres ensure the separation, due to the lose structure of the space. Due to the absence of significant branches across these, no vessel needs to be cut off; thus, causing damage. In addition, easy lateral extraction and the length of this space (average: $6.04 \mathrm{~cm}$ ) are responsible for the clear exposure of the corresponding parts of the elbow. Typically, these two vessels constitute the cubital articular rete, without nourishing specific muscle tissues; thus, having greater range for retraction. Moreover, it is located in the anterior elbow. Hence, elbow flexion and extension determine the large range for retraction. The extremely thin blood vessel branches on the surfaces between the brachialis can be retracted with a gauze, which generally does not cause bleeding. After the lateral retraction of the nerves and blood vessels through simple layers, the longitudinal split of the brachial muscle gives a perfect exposure of the trochlea and coronoid for ready front-to-back fracture fixation. This satisfactorily meets the requirements of the optimal front-to-back fixation proposed in medical literatures [2]. Hence, this approach is reasonable and feasible. According to our study on the lateral branches of the brachial artery and radial artery, as well as medial branches of the humeral artery and ulnar artery, lateral retraction of the vessels is safer and more reasonable; because blood vessel branches are rarely cut off, reducing damage. Studies on muscle branches of the median nerve provided a basis for tissue repair [12], while this study explored the distribution of the median nerve and its branches, providing anatomical support for surgical approach. The muscle branches were given off laterally from the trunk at a minimal acute angle, and were almost parallel to the trunk. These anatomical characteristics found in the present study are consistent with reports in literature $[3,14,18]$. We did not find articular branches of the median nerve with the anterior approach, which accorded with the anatomical feature where articular branches of the median nerve were given off dorsally [6]. This makes medial retraction of the median nerve reasonable. Many scholars [19] have studied anatomical features of specific lengths of muscular branches in the median nerve, as well as the branches; which also proves the safety of medial retraction of the median nerve.

\section{Advantages, indications and precautions of the anterior neurovascular approach to the elbow}

The anterior neurovascular interval approach to the elbow has numerous advantages, including simple anatomical layer, good exposure, strong operability under direct visualisation, large range for retraction of the nerves and blood vessels, neurovascular protection under direct visualisation, less damage, less soft tissue dissection and no ectopic ossification, easy restoration of joint function and low incidence of complications of the elbow, as well as safety, rationality and feasibility. This approach has wide indications, which involve the ulnar coronoid fracture, especially coronal shear fractures of the medial-distal humerus due to the perfect exposure of the coronal fossa of the humerus and trochlea, and can be applied in the treatment of terrible triad in combination with other surgical approaches, and in the relaxation of the anterior capsule in joint contracture. Although this approach has its own advantages, including the clear exposure of important structures such as nerves and blood vessels, and no damage, and similar to the radial nerve exposed in the humeral shaft fracture, which is less likely to be damaged under the protection, care must be taken in the surgical procedure. Most importantly, we must be clear about the local anatomical structure, which is the premise to reduce neurovascular injury. In the meanwhile, we must be familiar with other approaches, because sometimes a combined approach is needed to deal with a serious elbow injury. We should not make an incision across the elbow crease, so as not to affect elbow joint mobility due to scar contracture.

\section{The choice of the surgical approach to the elbow joint}

Good surgical approaches are necessary for a successful operation. Multiple surgical approaches have been described for coronoid repair [15]. A lateral approach to the elbow is a standard surgical method to gain access for contracture release and to treat a fracture on the lateral side of the elbow, such as the radial head and the capitellum [5]. Additionally, a lateral approach could be used to repair the lateral joint capsule and the lateral collateral ligament. The lateral approach is not preferable for an isolated fracture of the ulnar coronoid process. Consequently, a medial approach is recommended for the patients in fractures of the coronoid process, particularly for those with a varus or posteromedial rotational injury. 
With regard to internal fixation with a plate for an anteromedial facet fracture of the ulnar coronoid process, the medial approach is the best choice $[1,11]$. However, with the medial approach, it is difficult to expose the anterior part of the coronoid process; thus, a relatively long incision and more soft tissue dissection are required. The posterior approach is predominantly applicable to patients with ulnar olecranon fractures; however, exposure and fixation of the coronoid are prone to difficulties as well. An ulnar coronoid fracture alone is not treated through a posterior approach. The ideal surgical approach for an elbow fracture should minimise soft tissue dissection without compromising fracture visualisation and reduce the risk of heterotopic ossification $(\mathrm{HO})$ and elbow stiffness [14]. Peach and Stanley [13] provided a detailed description of the posterior, medial and lateral approach to the elbow with respect to the indications, contraindications, anatomy, specific operations, advantages and disadvantages; the anterior approach was excluded from the study. In this study, the anterior approach for fracture of an ulnar coronoid process fracture has its unique advantages, including a simple anatomical layer, wide exposure of the joint, easy mastery and application, minimal soft tissue dissection, and no injury to the normal anatomic structure of the elbow joint, including the ulnar flexor of the wrist, the ending point of the pronator teres, the ulnar collateral ligament and the ulnar nerve. Additionally, the advantages include minimal injury and an absence of $\mathrm{HO}$ as well as the avoidance of adverse outcomes caused by extensive soft tissue dissection, particularly $\mathrm{HO}$ [15]. The anterior approach provides a wide and clear operation field, direct vision for the open reduction, strong fixation caused by vertical compression of the fracture fragment, and early functional exercises. In this study, the proposed anterior approach is in accordance with other reports concerning skin incision; however, in terms of the specific tissue space, our procedure is different. In regard to an incision in the superficial layer of the deep fascia, other reports $[9,11]$ revealed that the brachial artery and median nerve are retracted to one side. We perform the incision in the space between the brachial artery and the median nerve. The separation is obtainable because of the loose space. The brachial artery has no medial branches; it has lateral branches to provide nutrition to the muscles, such as the brachioradialis and supinator muscles. The medi- an nerve has no lateral branches except the medial ones to control the muscles, including the pronation teres, flexor carpi radials and flexor digitorum superficial muscle. The gap between the two is very loose and can be separated and retracted easily. the median nerve has an extensive sliding range when the elbow joint is either flexed or extended. Some scholars [20] have questioned the report by Han et al. [9] that some arterial branches constitute a "vascular tree" and that the distance to the medially retracted brachial artery was constricted. On the other hand, an exposure of the blood vessels and nerves retracted laterally might damage the medial branches of the median nerve, resulting in neurological symptoms [21]. An incision in the space between the brachial artery and the median nerve is thus the optimal approach, reducing injury to the vascular and nerve branches.

\section{Limitations of this study}

The limitation of this study is the shortage of cadaver specimens. Due to the small number of specimens, variations in anterior median nerve, brachial artery and radial-ulnar artery, especially vascular variations and the small sample size, it is difficult to reveal its essence and regularity.

\section{CONCLUSIONS}

Based on the anatomical study of cadavers, the brachial artery and radial artery give off more thick lateral branches close to the ulna coronoid, while the brachial artery and ulnar artery give off fewer thin medial branches. The brachial artery and ulnar artery were found to have a long portion of the concomitant median nerve, without any large branches crossing these. The gap is very loose and can be easily separated. The lateral retraction of the brachial artery, ulnar artery, biceps tendon and distolateral brachioradialis, as well as the medial retraction of the median nerve and the pronator teres, provides excellent exposure of the brachialis. The longitudinal split and retraction of the brachial muscle clearly expose the anterior capsule, coronoid process of ulna, and the anterior side of the distal humerus.

Anatomical studies have found that this approach is optimal to expose the anatomical structures of the anterior side of the ulnar elbow for the surgical treatment of various fractures, such as ulna coronoid fractures, coronal shear fractures of the distal trochlear articular surface, and terrible triad. 


\section{REFERENCES}

1. Aksu N, Korkmaz MF, Göğüş A, et al. [Surgical treatment of elbow dislocations accompanied by coronoid fractures]. Acta Orthop Traumatol Turc. 2008; 42(4): 258-264, doi: 10.3944/aott.2008.258, indexed in Pubmed: 19060520.

2. An KN, Hui FC, Morrey BF, et al. Muscles across the elbow joint: a biomechanical analysis. J Biomech. 1981; 14(10): 659-669, doi: 10.1016/0021-9290(81)90048-8, indexed in Pubmed: 7334026.

3. Anne MA, Arthur FD. Grant. Anatomical Atlas. Translated by Zuo HC. 12th Ed. Shanghai Science and Technology Press, Shanghai 2011: 540.

4. Chantelot $C$, Feugas $C$, Guillem P, et al. Innervation of the medial epicondylar muscles: an anatomic study in 50 cases. Surg Radiol Anat. 1999; 21(3): 165-168, doi: 10.1007/ bf01630894, indexed in Pubmed: 10431328.

5. Cheung EV, Steinmann SP. Surgical approaches to the elbow. J Am Acad Orthop Surg. 2009; 17(5): 325-333, doi: 10.5435/00124635-200905000-00007, indexed in Pubmed: 19411644

6. Ding HM, Fan SQ, Yin ZX, et al. Anatomical studies on muscle branch of forearm median nerve. Prac Med Tech. 2006; 13: 4297-4298.

7. Elkowitz SJ, Polatsch DB, Egol KA, et al. Capitellum fractures: a biomechanical evaluation of three fixation methods. J Orthop Trauma. 2002; 16(7): 503-506, doi: 10.1097/00005131-200208000-00009, indexed in Pubmed: 12172281.

8. Gao SL. Practical Anatomical Atlas (Upper Limb).3rd Ed. Shanghai Science and Technology Press, Shanghai 2012: 152.

9. Han SH, Yoon HK, Rhee SY, et al. Anterior approach for fixation of isolated type III coronoid process fracture. Eur J Orthop Surg Traumatol. 2013; 23(4): 395-405, doi: 10.1007/ s00590-012-1007-y, indexed in Pubmed: 23412150.
10. Kong XY, Yang SZ. Local Anatomy, 1st Ed. World Book Publishing Company, Xi'an 2014: 152.

11. O'Driscoll SW, Jupiter JB, Cohen MS, et al. Difficult elbow fractures: pearls and pitfalls. Instr Course Lect. 2003; 52: 113-134, indexed in Pubmed: 12690844.

12. Pan $X D$, Liu WB, Shan YG. Anatomy and clinical significance of elbow branches of the median nerve. J Neck Waist Pain. 1993; 14: 155-156.

13. Peach C, Stanley D. Surgical approaches to the elbow. Orthop Trauma . 2012; 26(5): 297-302, doi: 10.1016/j. mporth.2012.09.008.

14. Ring D, Jupiter JB. Surgical exposure of coronoid fractures. Techn Shoulder Elbow Surg . 2002; 3(1): 48-56, doi: 10.1097/00132589-200203000-00010.

15. Shukla DR, Koehler SM, Guerra SM, et al. A novel approach for coronoid fractures. Tech Hand Up Extrem Surg. 2014; 18(4): 189-193, doi: 10.1097/BTH.0000000000000065, indexed in Pubmed: 25289725.

16. Standing S. Gray's Anatomy. Translated by Xu QY. 39th Ed. Peking University Medical Press, Peking 2008: 957-964.

17. Su XY, Tang PF. Anatomical and clinical study of surgical approach of the elbow. Chin J Anat Clin Med. 2015; 20: 276-280.

18. Tang ZW, Sun TK, Zhang SQ, et al. Test of muscular branches of upper limb nerves and distribution. Anatomy Newspaper. 1963; 6: 96-105.

19. Zhao BC, Yuan TX, Ma XL, et al. Anatomical and clinical study of anterior approach of the elbow in the treatment of ulna coronoid fractures. Chin J Orthop. 2015; 35: 859-864.

20. Zhu Xz, Wang X, Ma Z. Comment on the anterior approach for coronoid process fracture. Eur J Orthop Surg Traumatol. 2014; 24(1): 123-124, doi: 10.1007/s00590012-1124-7, indexed in Pubmed: 23412249.

21. Zuo YM, Wang ZQ, Wang YG. Treatment of fracture of ulna coronoid process. ChinJ Orthop. 2006; 26: 366-370. 